\title{
Iatrogenic atrioventricular bypass tract following a Fontan operation for tricuspid atresia
}

\author{
Eric Rosenthal, Julian Bostock, Jaswinder Gill
}

Guy's Hospital, London: Department of Paediatric Cardiology E Rosenthal J Bostock

Department of Adult Cardiology

J Gill

Correspondence to:

Dr E Rosenthal, Department

of Paediatric Cardiology,

of Paediatric Cardiology,
Guy's Hospital, St Thomas

St, London SE1 9RT.

Accepted for publication 25 October 1996

Figure 1 (A) The right atrial angiogram (right anterior oblique projection) shows the imperforate right atrioventricular connection (arrow) and the

anastomosis between the right atrial appendage and the right ventricular outflow tract (RVOT) (arrow heads). (B) The right ventricular angiogram shows a moderate sized right ventricle $(R V)$ and the imperforate right atrioventricular connection (arrow). (C) An electrode recording a His bundle electrogram (His); a coronary sinus catheter coronary sinus catheter
(CS), and the ablation catheter $(A)$ in the anastomosis between the right atrial appendage and the right ventricular outflow tract are shown in the same projection. An additional electrode catheter is in the right ventricular outflow tract. The effective ablation site is shown in (D). $R A$, right atrium; $R P A$, right pulmonary artery.

\begin{abstract}
A 16 year old female with tricuspid atresia had undergone a Fontan operation at four years of age. Two years later she first presented with a narrow complex tachycardia which could only be partially controlled on flecainide in high doses. On electrophysiological study, the tachycardia was found to be due to atrioventricular re-entry within the surgical right atrial to right ventricular outflow tract anastomosis. Radiofrequency ablation at this site abolished the arrhythmia and she is now symptom-free on no medication.
\end{abstract}

(Heart 1997;77:283-285)

Keywords: Fontan operation; supraventricular tachycardia; radiofrequency ablation
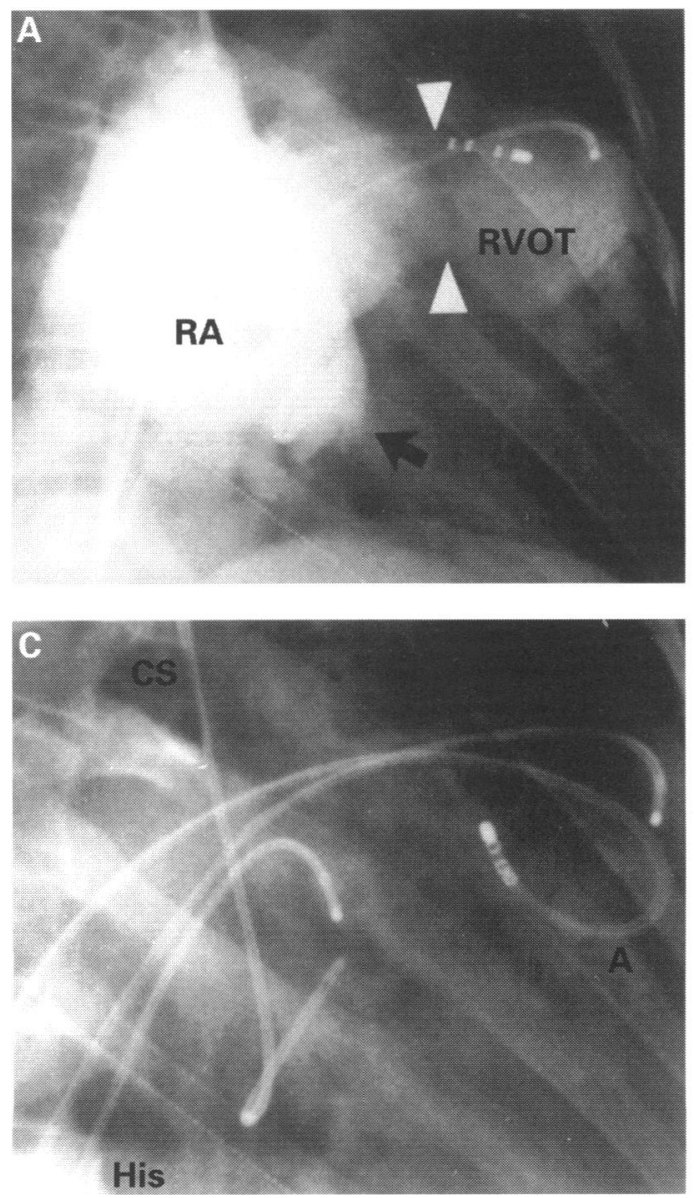

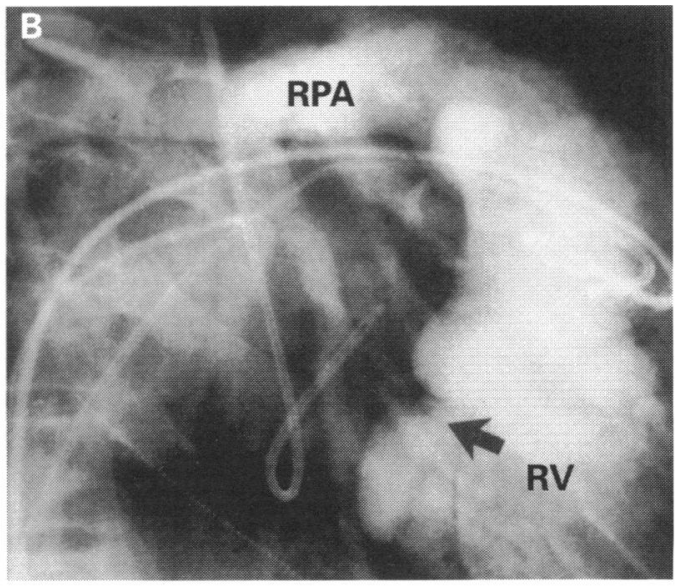

\section{Case report}

A 16 year old female was admitted for electrophysiological study with a view to radiofrequency ablation for persistent supraventricular tachycardia. At the age of three months she had presented in heart failure and was found to have tricuspid atresia with a non-restrictive ventricular septal defect, concordant ventriculo-arterial connections, and a large patent arterial duct. Following duct ligation she was no longer in heart failure and she underwent a Fontan operation at age four years. The right atrial appendage was anastomosed to the right ventricular outflow tract and the ventricular septal defect was closed.

She remained well for two years and then presented with vomiting and non-specific symptoms and was found to have a supraventricular tachycardia of 240 beats $/ \mathrm{min}$.

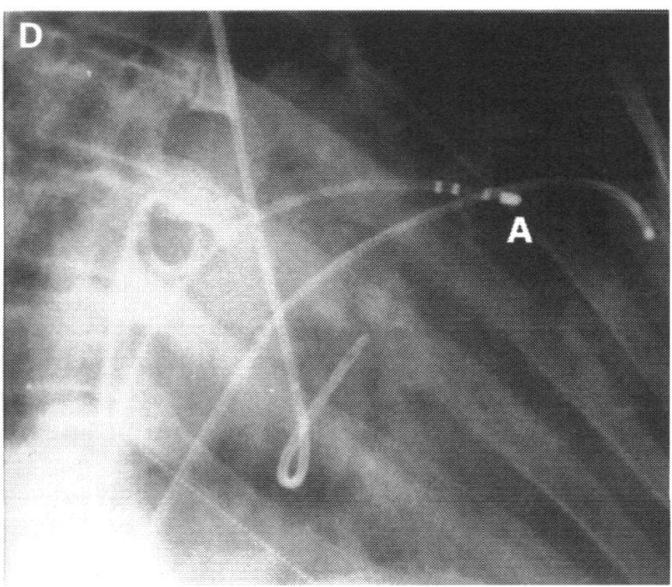


A

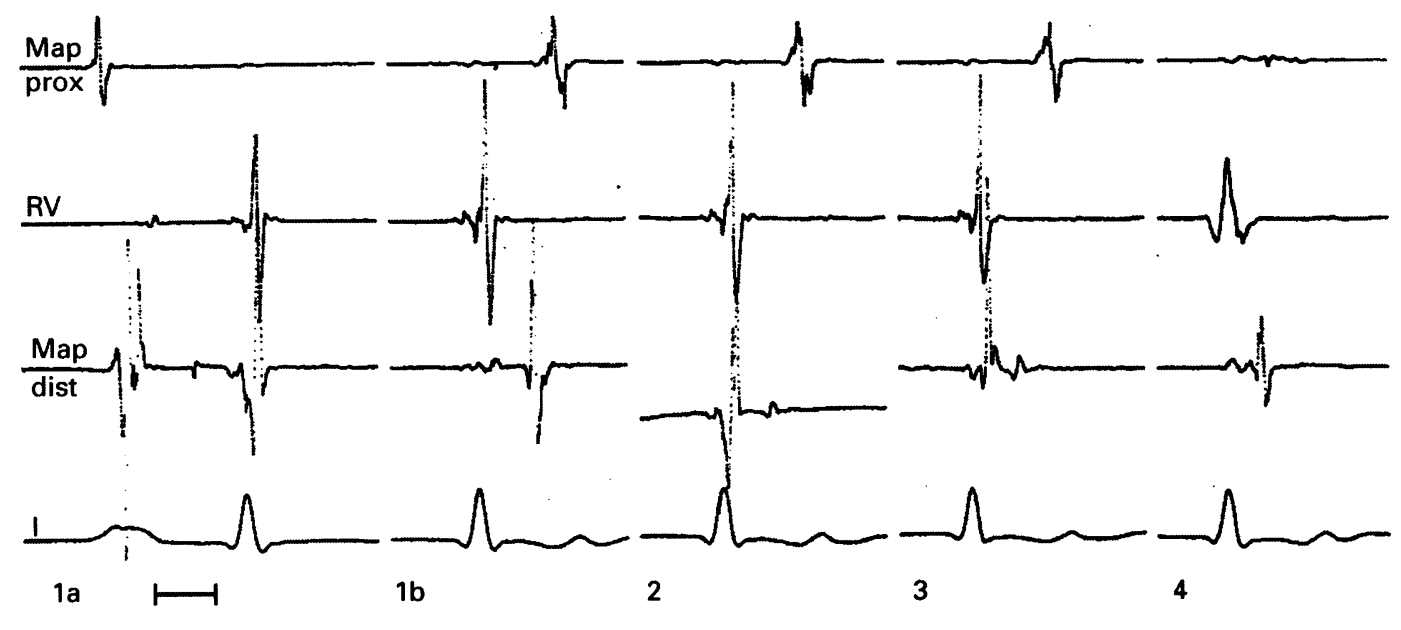

Figure 2 Electrograms recorded via the mapping catheter, together with surface lead I and right ventricular electrogram are shown in $(A)$. Mapping catheter locations are illustrated in $(B) .1=H$ is position in right atrium during sinus rhythm (1a) and in tachycardia (1b) electrograms in 2, 3, and 4 are during tachycardia: $2=$ left ventricular side of mitral valve; $3=$ right ventricular side of right atrioventricular junction; $4=$ ablation site in superior margin of right atrial appendage to right ventricular outflow tract anastamosis (arrow heads). I, surface lead I; Map prox, proximal poles of mapping catheter; Map dist, distal poles of mapping catheter; $R V$, right ventricle; $L V$, left ventricle; $R A$, right atrium; MPA, main pulmonary artery. Time marker is atrum

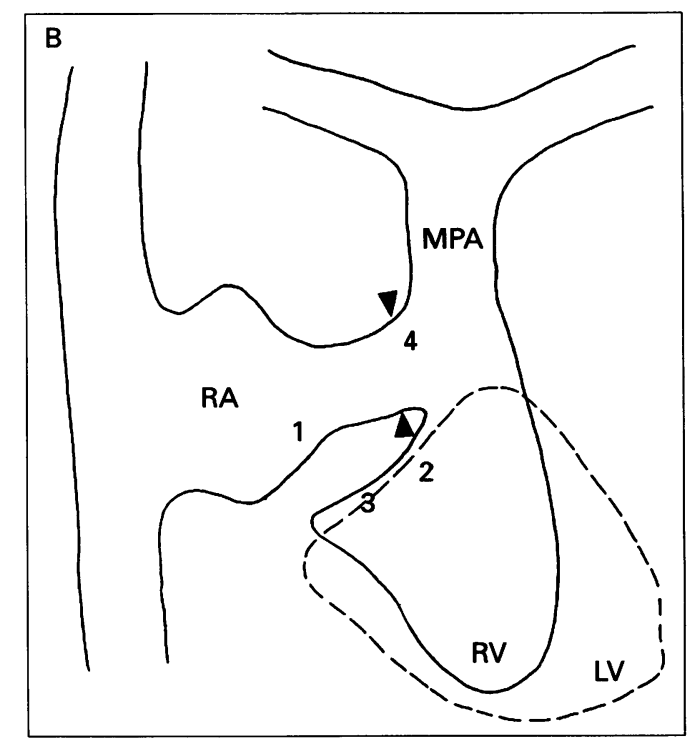

Intravenous verapamil was ineffective and following oral digitalisation, intravenous disopyramide converted her to sinus rhythm. An electrophysiological study confirmed that the arrhythmia was due to atrioventricular reentry, and intravenous flecainide abolished the supraventricular tachycardia. She was discharged on oral flecainide with moderate control and had intermittent breakthrough tachycardias despite a high serum flecainide level $(900 \mathrm{ng} / \mathrm{l})$. She was therefore admitted for treatment of her arrhythmia by radiofrequency ablation.

Multiple electrode catheters were placed in the high right atrium, low right atrium to record a His bundle signal, right ventricle through the Fontan anastomosis, and the left ventricle. An angiographic catheter was placed in the coronary sinus as a radiographic landmark from a subclavian vein puncture. In the presence of tricuspid atresia the working diagnosis was a concealed left sided atrioventricular bypass tract. Tachycardia was easily initiated and terminated with paired atrial or ventricular extrastimuli and terminated by adenosine. During tachycardia, mapping in the left ventricle around the mitral valve annulus did not provide evidence of a left sided bypass tract. Angiography showed that the atretic right atrio-

ventricular valve was of a significant size (fig 1A, B) and initial mapping in both the right atrium and right ventricle, on either side of the atretic tricuspid valve, suggested a pathway at the superior portion of the atretic tricuspid valve (fig $2 \mathrm{~A}$ ). Test applications in this area were ineffective. Subsequently mapping of the right atrium to right ventricular anastomosis showed the shortest ventriculo-atrial conduction times on the superior portion of the anas- $N$ tomosis (fig $2 \mathrm{~A}, \mathrm{~B}$ ). RF delivery at this site (fig 1D), using a 7F RF Marinr catheter (Medtronic, San Jose, California) powered by an Atakr generator (Medtronic), abolished tachycardia and ventriculo-atrial conduction. She was discharged the following day off all antiarhythmic drugs and had remained symptom-free for two months at the time of writing.

\section{Discussion}

Atrial flutter and atrial ectopic tachycardia after a Fontan type operation may be related to the surgical dissection around the sinoatrial node and atrium, or the right atrial distension that occurs following surgery, or both. It often indicates a poor haemodynamic result. ${ }^{12} \mathrm{Re}$ entry tachycardias related to bypass tracts or atrioventricular-nodal re-entry are less com- 
mon and are presumably related to preexisting anatomical connections during development.

In our patient, who had an excellent haemodynamic result, the appearance of an atrioventricular re-entry tachycardia was presumed to be due to activation of a concealed accessory pathway. Intravenous flecainide was effective, as was initial oral treatment, but subsequently she required high serum levels, producing only poor control. Together with concerns about possible risks of flecainide it was decided to attempt radiofrequency ablation. In tricuspid atresia the atrioventricular connection on the right is absent; the atrioventricular junction is therefore much reduced and it was assumed that a left sided pathway was the probable substrate for the arrhythmia. Angiography at the time of the procedure suggested that the tricuspid atresia may have been due to an imperforate valve with a rather more substantial junction than first anticipated. Mapping of both the left and right atrioventricular junctions did not reveal a pathway. Mapping in the area of the right atrial appendage to right ventricular outflow tract anastomosis showed the site of the bypass tract, which was successfully ablated.

Conduction across the atrio-atrial anastomosis has been documented after cardiac transplantation and appears to be due to growth of excitable tissue across the surgical scar. ${ }^{34}$ There is a previous report of ablation after the Fontan operation where the authors describe the successful ablation site to be in the superior portion of the atretic tricuspid valve. ${ }^{5}$ They thought the bypass tract was congenital in origin. From their description, however, and from the evidence in this case, it is possible that the substrate in their patient was also the growth of excitable tissue across the surgical scar.

Growth of excitable tissue across a surgical scar may be possible in patients who have undergone this form of the Fontan operation. Currently the surgical approach is to perform a direct atrial to pulmonary artery anastomosis with an intra-atrial patch, to channel inferior caval blood to the pulmonary artery. Thus the occurrence of this complication should not occur in patients from the present surgical era.

1 Gewillig M, Wyse RK, de Leval MR, Deanfield JE. Early and late arrhythmias after the Fontan operation: predisand late arrhythmias after the Fontan operation: predis-
posing factors and clinical. Br Heart $\mathcal{f}$ 1992;67:72-9.

posing factors and clinical. Br Heart f $1992 ; 67: 72-9$.
2 Peters NS, Somerville J. Arrhythmias after the Fontan procedure. Br Heart $₹$ 1992;68:199-204.

3 Anselme F, Saoudi N, Redonnet M, Letac B. Atrioatrial conduction after orthotopic heart transplantation. $\mathcal{F} \mathrm{Am}$ Coll Cardiol 1994;24:185-9.

4 Rothman SA, Miller JM, Hsia HH, Buxton AE. Radiofrequency ablation of a supraventricular tachycardia due to interatrial conduction from the recipient to donor atria in an orthotopic heart transplant recipient. $\mathcal{F}$ Cardiovasc Electrophysiol 1995;6:544-50.

5 Case CL, Schaffer MS, Dhala AA, Gillette PC, Fletcher SE. Radiofrequency catheter ablation of an accessory atrioventricular connection in a Fontan patient. Pacing Clin Electrophysiol 1993;16:1434-6. 